\title{
Research on Nonlinear Dynamic Characteristics and Stability of Aerodynamic Bearings
}

\author{
Jia Chen-Hui*, Du Cai-Feng and Qiu Ming
}

School of Mechatronics Engineering, Henan University of Science and Technology, Xiyuan Road 48, Box 62, Luoyang, Henan, 471003, China

\begin{abstract}
In order to research the conical spiral groove aerodynamic bearings, the lubrication mathematical model of the bearings was established. The Reynolds equation of the laminar flow condition is used to calculate the partial differential equation of the perturbation pressure with the local finite difference method. Through calculating the stiffness and damping coefficient, the influence of the speed of law and eccentricity ratio on the dynamic characteristic coefficients has been gained. The mathematical model for the stability of the bearing-rotor system is established to study the influence law of speed influence of the law of speed and eccentricity ratio on the stability. The results show that the influence of the bearing's speed and eccentricity on the dynamic characteristics is significant. A reasonable choice of the bearing's speed and eccentricity contributes to improve the dynamic characteristics and the stability of the bearing-rotor system.
\end{abstract}

Keywords: Aerodynamic bearing, Dynamic stiffness coefficients, Dynamic damping coefficients, Nonlinear dynamic characteristics, Stability, Perturbation Pressure.

\section{INTRODUCTION}

Aerodynamic bearing depends on the gas dynamic pressure film that has formed in the bearing clearance with a certain stiffness to support the rotor. The advantages are that it does not require external air feeder, with low friction, high speed and long service life, etc. Based on the above advantages, the aerodynamic bearings are widely used in precision mechanic, space technology, medical equipment and other fields $[1,2]$. The major disadvantages are low load capacity, small stiffness and poor stability.

With the development of high speed and ultra-high speed technology, aerodynamic bearings are widely used in high speed rotating machinery. Due to the high rotation speed of the bearing that can reaches up to hundreds of thousands of revolutions per minute, the gas film pressure changes is a very complicated nonlinear stochastic process $[3,4]$. When subjected to the influence of external factors, the rotor will perform random movement with the load variation. The behavior of the rotor and the stability of the bearing-rotor system are affected directly by the bearing's dynamic characteristics $[5,6]$. If the bearing-rotor system is not stable, the rotor bearing capacity will not be stable which would cause the rotor to deviate from the steady-state position, potentially resulting in increased friction and/or collision to damage the bearing.

Therefore, studying the bearing's static characteristics alone is not sufficient to meet the requirements for the practical application of the bearing. It is necessary to

\footnotetext{
*Address correspondence to this author at the School of Mechatronics Engineering, Henan University of Science and Technology, Xiyuan Road 48, Box 62, Luoyang, Henan, 471003, China; Tel:0086-13523798226; E-mail: xjiachenhui@163.com
}

research the bearing's dynamic characteristics in-depth, and to analyze the stability of the bearing-rotor system.

\section{THEORETICAL ANALYSIS}

\subsection{Fundamental Bearing Layout}

The cone spiral groove aerodynamic bearing is mainly composed of rotor and stator. The surface of the rotor is machined on a certain number of spiral grooves. The spiral groove consists of a ridge and a groove; the fundamental bearing layout is illustrated in Fig. (1). As the rotor rotates
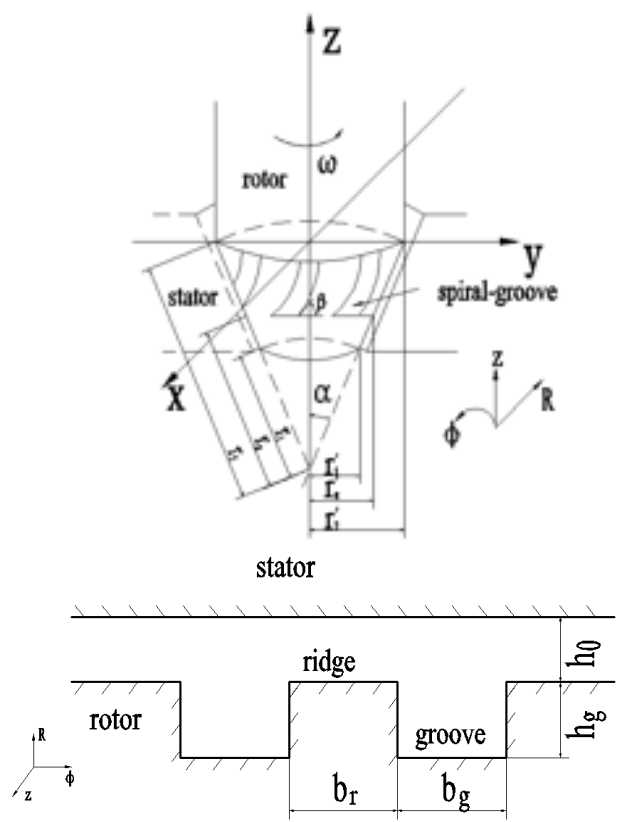

Fig. (1). Conical spiral groove aerodynamic bearing. 
relative to the stator at a high speed, the viscous gas is brought into the wedge-shaped clearance, in which the dynamic pressure effect is formed. Then, the gas film pressure is produced and the dynamic pressure suspension is formed. The spiral groove not only forms the staircase effect in the bearing clearance, but also enhances the pumping effect and promotes the dynamic pressure effect.

\subsection{Mathematical Modeling}

Based on the computational fluid dynamics, the gas lubrication theory, the fluid motion equation, the continuity equation and the ideal gas equation have been established [79]. The nonlinear dimensionless Reynolds equation of conical spiral groove aerodynamic bearings under the state of laminar flow is established in the transient state. Using the conformal transformation, the solution domain is turned into a standard rectangular [10-12]. The conversion formula can be expressed as:

$$
\begin{aligned}
& \psi=\ln R / \sin \alpha, d \psi=d R /(R \sin \alpha) \\
& \frac{\partial}{\partial \phi}\left(H^{3} \frac{\partial P^{2}}{\partial \phi}\right)+\frac{\partial}{\partial \psi}\left(H^{3} \frac{\partial P^{2}}{\partial \psi}\right)=2 \Lambda \gamma \sin ^{2} \alpha \frac{\partial(P H)}{\partial \phi} \\
& \quad+\left(h_{0} \omega \varepsilon_{1}^{\prime} \cos \phi+e \omega \theta^{\prime} \sin \phi\right) \cos \alpha-h_{0} \omega \varepsilon_{2}^{\prime} \sin \alpha
\end{aligned}
$$

where, $\alpha$ is the cone angle, $h_{0}$ is the bearing clearance, $\omega$ is the rotational speed, $p$ is the gas film pressure, $\phi$ is the circumferential direction coordinate, $R=r / r_{1}$ is the dimensionless radial radius, $H=h / h_{0}$ is the dimensionless film thickness, $P=p / p_{a}$ is the dimensionless gas film pressure, $\varepsilon_{1}=e_{1} / h_{0}$ is the dimensionless radial eccentricity, $\varepsilon_{2}=e_{2} / h_{0}$ is the dimensionless axial eccentricity, $\theta^{\prime}$ is the dimensionless rotational speed, $\theta$ is the position angle, $\Lambda=6 \mu \omega r_{1}^{2} / P_{a} h_{0}^{2}, d \psi=d R /(R \sin \alpha)$.

\section{SOLUTION OF DYNAMIC CHARACTERISTIC COEFFICIENTS}

\subsection{The Stiffness and Damping Equations}

If the rotor deviates from the steady-state position $O_{1}$ in the $\xi, \eta$ and $z$ directions, as shown in Fig (2), the gas pressure of the steady-state position can be expressed as:

$p_{0}=p(\psi, \varphi, Z ; e, \theta, z ; 0,0,0)$

The gas pressure of the transient state position can be expressed as:

$p=p\left(\psi, \varphi, Z ; e, \theta, z ; e^{\prime}, e \theta^{\prime}, z^{\prime}\right)$

The transient pressure $p$ can be expanded into Taylor series that the rotor deviates from the steady-state position (omitted the second order term).

$$
\begin{aligned}
p= & p_{0}+p_{e} \Delta e+p_{\theta} \Delta(e \Delta \theta)+p_{z} \Delta z \\
& +p_{e^{\prime}} \Delta e^{\prime}+p_{\theta^{\prime}} \Delta\left(e \Delta \theta^{\prime}\right)+p_{z^{\prime}} \Delta z^{\prime}
\end{aligned}
$$

where, $\left(\Delta e=\left(e-e_{0}\right), e \Delta \theta=e \theta-e \theta_{0}, \Delta z=z-z_{0}\right)$ are transient displacement; $\left(\Delta e^{\prime}, e \Delta \theta^{\prime}, \Delta z^{\prime}\right)$ are transient speed; $\left(p_{e}, p_{\theta}, p_{z}, p_{e^{\prime}}, p_{\theta^{\prime}}, p_{z^{\prime}}\right)$ are the variation rate that the $p$ changes with $\left(\Delta e, e \Delta \theta, \Delta z, \Delta e^{\prime}, e \Delta \theta^{\prime}, \Delta z^{\prime}\right)$ which is called the perturbation pressure.
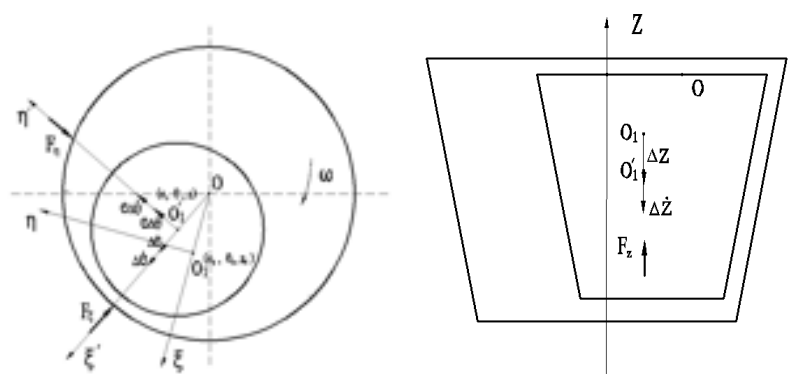

Fig. (2) Force analysis.

The gas force for whirling slightly can be derived:

$$
\left.\begin{array}{c}
F_{e} \\
F_{\theta} \\
F_{z}
\end{array}\right\}=-\int_{r_{0}}^{r_{2}} \int_{\varphi_{1}}^{\varphi_{2}} p\left\{\begin{array}{c}
\sin \alpha \cos \alpha \cos \varphi \\
\sin \alpha \cos \alpha \sin \varphi \\
\sin ^{2} \alpha
\end{array}\right\} r d d \varphi
$$

The gas film force derivative of the transient displacement is known as the gas film stiffness coefficient $k_{i j} . i$ is the direction of the force increment and $j$ is the direction of the displacement increment.

$$
\begin{aligned}
& k_{e e}=\left(\frac{\partial F_{e}}{\partial e}\right)_{0} ; k_{e \theta}=\left(\frac{\partial F_{e}}{e \partial \theta}\right)_{0} ; k_{e z}=\left(\frac{\partial F_{e}}{\partial z}\right)_{0} \\
& k_{\theta e}=\left(\frac{\partial F_{\theta}}{\partial e}\right)_{0} ; k_{\theta \theta}=\left(\frac{\partial F_{\theta}}{\partial \theta}\right)_{0} ; k_{\theta z}=\left(\frac{\partial F_{\theta}}{\partial z}\right)_{0} \\
& k_{z e}=\left(\frac{\partial F_{z}}{\partial e}\right)_{0} ; k_{z \theta}=\left(\frac{\partial F_{z}}{e \partial \theta}\right)_{0} ; k_{z z}=\left(\frac{\partial F_{z}}{\partial z}\right)_{0}
\end{aligned}
$$

The gas film force derivative of the transient speed is known as the gas film damping coefficient $b_{i j} . i$ is the direction of the force increment and $j$ is the direction of the displacement increment.

$$
\begin{aligned}
& b_{e e}=\left(\frac{\partial F_{e}}{\partial e^{\prime}}\right)_{0} ; b_{e \theta}=\left(\frac{\partial F_{e}}{e \partial \theta^{\prime}}\right)_{0} ; b_{e z}=\left(\frac{\partial F_{e}}{\partial z^{\prime}}\right)_{0} ; \\
& b_{\theta e}=\left(\frac{\partial F_{\theta}}{\partial e^{\prime}}\right)_{0} ; b_{\theta \theta}=\left(\frac{\partial F_{\theta}}{e \partial \theta^{\prime}}\right)_{0} ; b_{\theta z}=\left(\frac{\partial F_{\theta}}{\partial z^{\prime}}\right)_{0} ; \\
& b_{z e}=\left(\frac{\partial F_{z}}{\partial e^{\prime}}\right)_{0} ; b_{z \theta}=\left(\frac{\partial F_{z}}{e \partial \theta^{\prime}}\right)_{0} ; b_{z z}=\left(\frac{\partial F_{z}}{\partial z^{\prime}}\right)_{0} ;
\end{aligned}
$$

$\frac{\partial F_{i}}{\partial s_{j}}=-\frac{\partial}{\partial s_{j}} \int_{r_{0}}^{r_{2}} \int_{\varphi_{1}}^{\varphi_{2}} p f_{i} r d r d \varphi$

The derivative of Eq. (3) is solved, then the dimensionless stiffness coefficient $K_{i, j}=k_{i, j} /\left(p_{a} r_{1}^{2}\right)$ and the non-dimensional damping coefficients $B_{i, j}=b_{i, j} /\left(p_{a} r_{1}^{2}\right)$ can be derived: 


$$
\begin{aligned}
& \left.\begin{array}{c}
K_{e i} \\
K_{\theta i} \\
K_{z i}
\end{array}\right\}=-\int_{R_{0}}^{R_{2}} \int_{\phi_{1}}^{\phi_{2}} P_{j}\left\{\begin{array}{c}
\sin \alpha \cos \alpha \cos \phi \\
\sin \alpha \cos \alpha \sin \phi \\
\sin ^{2} \alpha
\end{array}\right\} R d R d \phi \\
& \text { Letting }(i, j)=\left(e, \varepsilon_{1}\right),(\theta, \theta),\left(z, \varepsilon_{2}\right) ; \\
& \left.\begin{array}{l}
B_{e i} \\
B_{\theta i} \\
B_{z i}
\end{array}\right\}=-\int_{R_{0}}^{R_{2}} \int_{\phi_{1}}^{\phi_{2}} P_{j}\left\{\begin{array}{c}
\sin \alpha \cos \alpha \cos \phi \\
\sin \alpha \cos \alpha \sin \phi \\
\sin ^{2} \alpha
\end{array}\right\} R d R d \phi \\
& \text { Letting }(i, j)=\left(e, \varepsilon_{1}^{\prime}\right),\left(\theta, \theta^{\prime}\right),\left(z, \varepsilon_{2}^{\prime}\right) ; \\
& \text { With } P_{\varepsilon_{1}}=\left(\frac{\partial P}{\partial \varepsilon_{1}}\right)_{0}, P_{\theta}=\left(\frac{1}{e} \frac{\partial P}{\partial \theta}\right)_{0}, P_{\varepsilon_{2}}=\left(\frac{\partial P}{\partial z}\right)_{0}, ; \\
& P_{\varepsilon_{1}^{\prime}}=\left(\frac{\partial P}{\partial \varepsilon_{1}^{\prime}}\right)_{0}, P_{\theta^{\prime}}=\left(\frac{1}{e} \frac{\partial P}{\partial \theta^{\prime}}\right)_{0}, P_{\varepsilon_{2}^{\prime}}=\left(\frac{\partial P}{\partial \varepsilon_{2}^{\prime}}\right)_{0}
\end{aligned}
$$

\subsection{Solution of Perturbation Pressure}

The difference expression of the governing equation Eq. (1) is established in the oblique coordinate system. The grids are meshed in the oblique coordinate system $(x=\phi-\psi / \operatorname{tg} \beta, y=\psi / \sin \beta$ ), its line should coincide with the boundary of the groove and ridge. The solution domain is meshed in the direction of $\mathrm{X}$ and $\mathrm{Y}$, as shown in Fig. (3). The range of $0 \sim 2 \pi$ is divided into $m$ grids in the $X$ direction, the net width is $f_{(A \cup B)_{i j}} ; i$ grids are divided in the $\mathrm{Y}$ direction, the net width is $l_{j}$. Furthermore, the grid nodes are numbered. $s_{(A \cup B)_{i j}}$ is the node number in the $\mathrm{X}$ direction, $i$ is the node number in the $\mathrm{Y}$ direction.

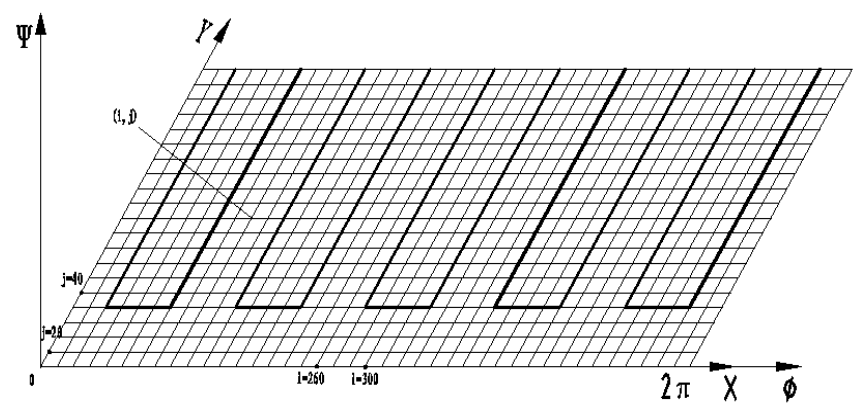

Fig. (3). Mesh generation.

The boundary conditions of the numerical calculation are given as follows:

Pressure boundary condition:

$P\left(r_{1}\right)=P\left(r_{2}\right)=1$

Symmetrical boundary condition:

$\left.\frac{\partial P}{\partial \varphi}\right|_{\varphi}=\left.\frac{\partial P}{\partial \varphi}\right|_{\varphi+2 \pi}$

The governing difference equation can be solved by Successive Over Relaxation. The convergence criterion is given by:
$\sum_{j=1}^{M} \sum_{i=1}^{N}\left|P_{i, j}^{(n)}-P_{i, j}^{(n-1)}\right| / \sum_{j=1}^{M} \sum_{i=1}^{N} P_{i, j}^{(n)} \leq \varepsilon$

where, $\varepsilon$ is 0.003 .

The perturbation pressure can be obtained by calculating the derivatives of Eq. (1) on the $\left(\varepsilon_{1}, \theta, \varepsilon_{2}, \varepsilon_{1}^{\prime}, \theta^{\prime}, \varepsilon_{2}^{\prime}\right) .\left(P_{\theta}, P_{\varepsilon_{2}}, P_{\varepsilon_{1}^{\prime}}, P_{\theta^{\prime}}, P_{\varepsilon_{2}^{\prime}}\right)$ can be solved.

Using $\mathrm{C}++$ is used to solve the differential equations and analyze the dynamic characteristic coefficients under different speed and eccentricity. The structural parameters and the operation parameters are shown in Table $\mathbf{1}$.

The flow chart of the numerical calculation is shown in Fig. (4):

\subsection{Numerical Analysis}

Fig. (5) shows the variation law of the calculated values of the dynamic characteristic coefficients with the speed $(\mathrm{n}=5000,10000 \mathrm{r} / \mathrm{min})$ and the eccentricity

$\left(\varepsilon_{2}=0.1,0.2,0.3,0.4,0.5,0.6\right)$.

Fig. (5a-c) shows the variation law of the calculated values of the bearing stiffness coefficients:

1) Due to the increase of the speed $n$, the dynamic pressure effect of the bearing increases gradually. It is found that the stiffness coefficients increase with the increase of the speed $n$.

2) In addition to the stiffness coefficient $K_{e \theta}$, the stiffness coefficients of aerodynamic bearing increase with the increase of the eccentricity $\varepsilon_{2}$.

3) As the main support directions $\xi, z$ of the load capacity, the value of the direct stiffness coefficients $K_{e e}$ and $K_{\mathrm{zz}}$ increases. While the value of the direct stiffness coefficient $K_{\theta \theta}$ is as small as the $\eta$ is the secondary support direction, and differs from the main support directions by an order of magnitude. The cross-coupled stiffness coefficients $K_{e \theta}, K_{\theta e}$, $K_{z \theta}$ and $K_{\theta z}$ relate to the secondary support directions a little less than the cross-coupled stiffness coefficients $K_{e z}$ and $K_{z e}$.

Fig. (5d-f) shows the variation law of the calculated values of the bearing damping coefficients:

1) Due to the increase of the speed $n$, the dynamic pressure effect of the bearing increases gradually. It is found that the damping coefficients increase with the increase of the speed $n$.

2) The damping coefficients of aerodynamic bearing increase with the increase of the eccentricity $\varepsilon_{2}$. While compared with the variation of the stiffness coefficients, the damping coefficients increase eases up. 


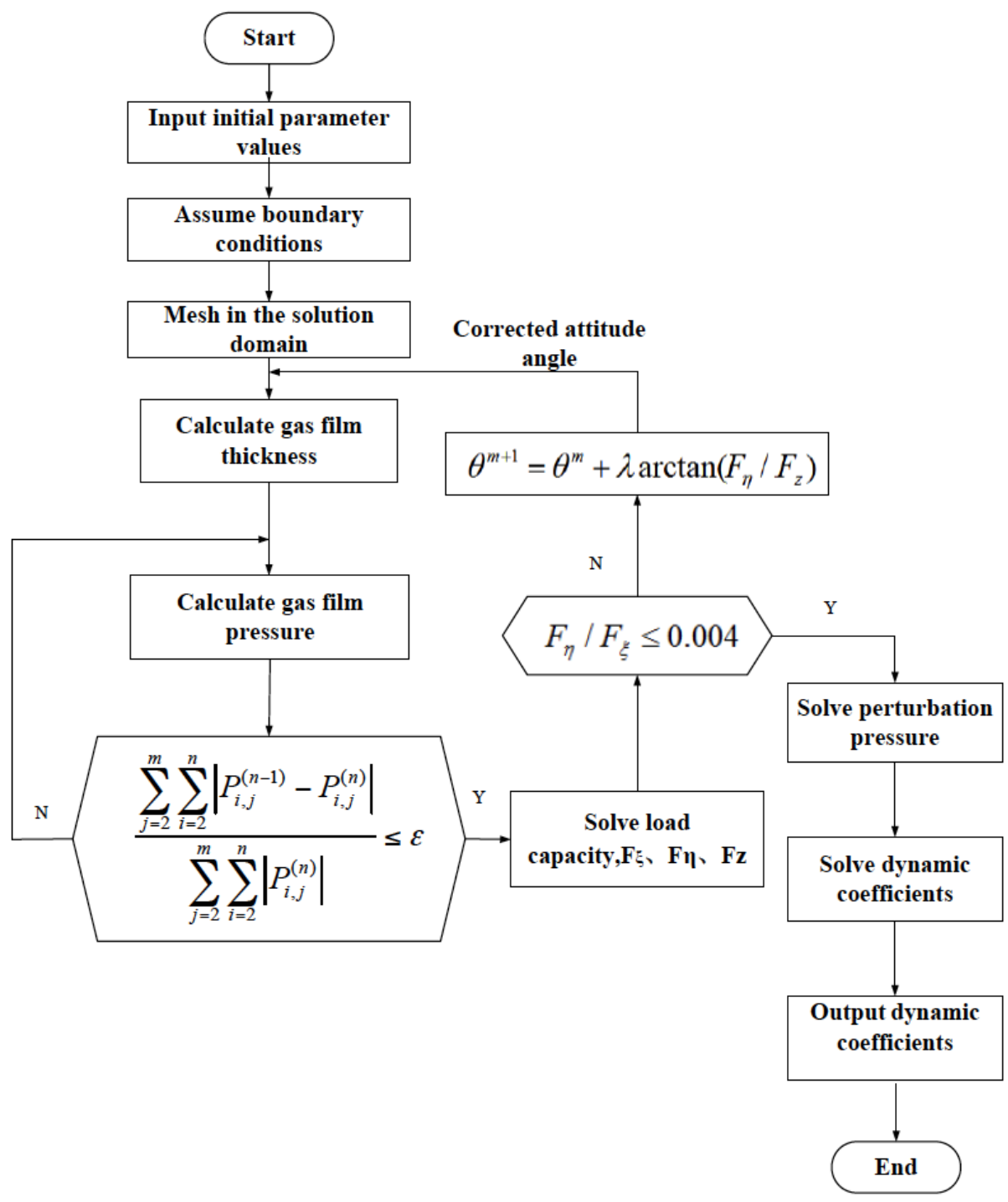

Fig. (4). Flow chart of numerical calculation.

3) As the main support directions $\xi, z$ of the load capacity, the value of the direct damping coefficients $B_{e e}$ and $B_{\mathrm{zz}}$ are large. While the value of the direct damping coefficient $B_{\theta \theta}$ is small as the $\eta$ is the secondary support direction. The cross-coupled damping coefficients $B_{e \theta}, B_{\theta e}, B_{z \theta}$ and $B_{\theta z}$ relate to the secondary support directions a little less than the cross-coupled damping coefficients $B_{e z}$ and $B_{z e}$.

\subsection{Experimental Analysis}

The dynamic characteristic coefficients, stiffness and damping are collected in the axial and radial directions. Then the experimental data is compared with the theoretical result. Based on the theoretical analysis and numerical analysis, the dynamic characteristic of the conical spiral groove aerodynamic bearings are experimented under the condition of different rotating speed and eccentricity. The dynamic characteristic coefficients of bearing in three main directions are obtained under the experimental conditions, which can provide the realistic foundation to improve the design and increase the performance and the capacity of gas bearings. Fig. (6) shows the variation law of the experimental values of the direct coefficients with the speed $(n=5000,10000 / \mathrm{min})$ and the eccentricity $\left(\varepsilon_{2}=0.1,0.2,0.3,0.4,0.5,0.6\right)$

1) The direct stiffness and damping coefficients increase with the increase of the speed and the eccentricity.

2) As the main support directions $\xi, z$ of the load capacity, the value of the direct stiffness coefficients $K_{e e}$ and $K_{\mathrm{zz}}$, and the direct damping coefficients $B_{e e}$, and $B_{\mathrm{zz}}$, increase. While the value of the direct stiffness coefficient $K_{\theta \theta}$ and the direct damping coefficient $B_{\theta \theta}$ is small as the $\eta$ is the non-main support direction.

3) The variations of the fitting curve are consistent. By comparing the values with the experimental values, the order of magnitude of the values is same. The 
(a) $K_{e e}, K_{\theta e}, K_{z e}$

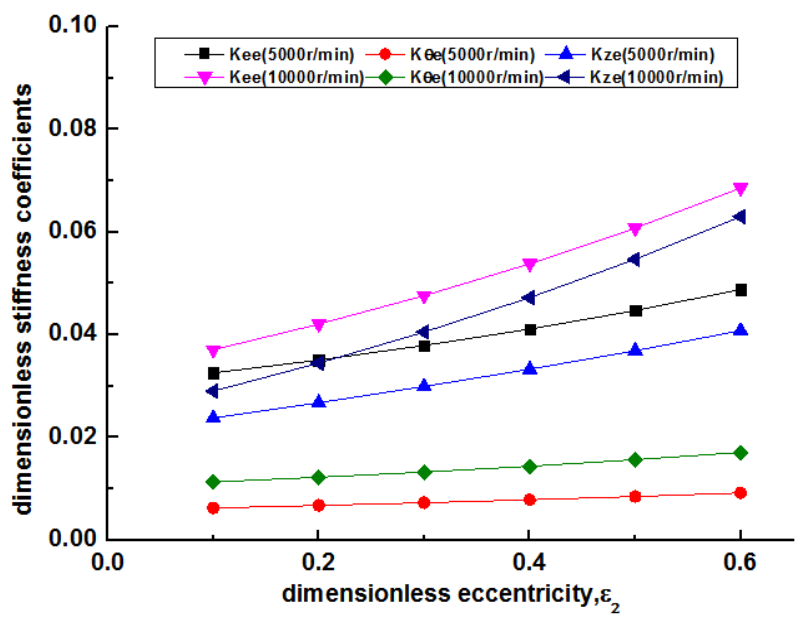

(c) $K_{e z}, K_{\theta z}, K_{z z}$

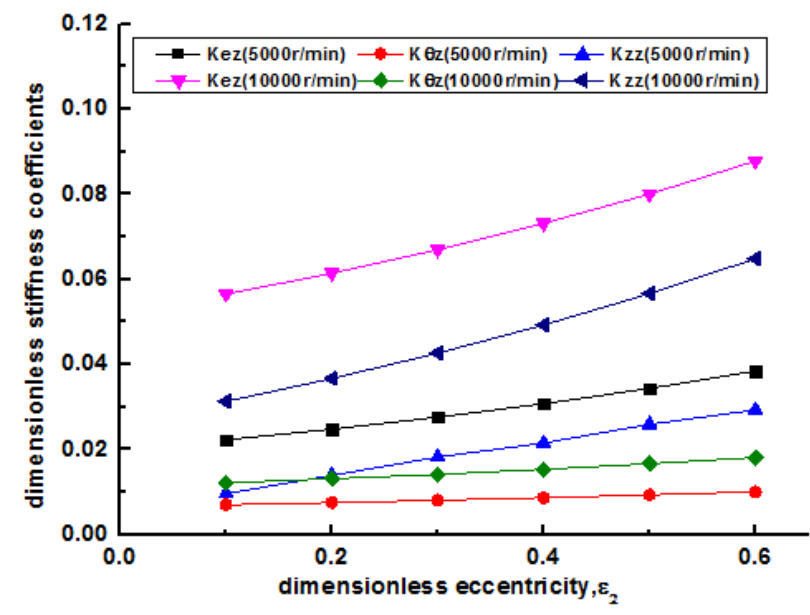

(c) $B_{e \theta}, B_{\theta \theta} B_{z \theta}$

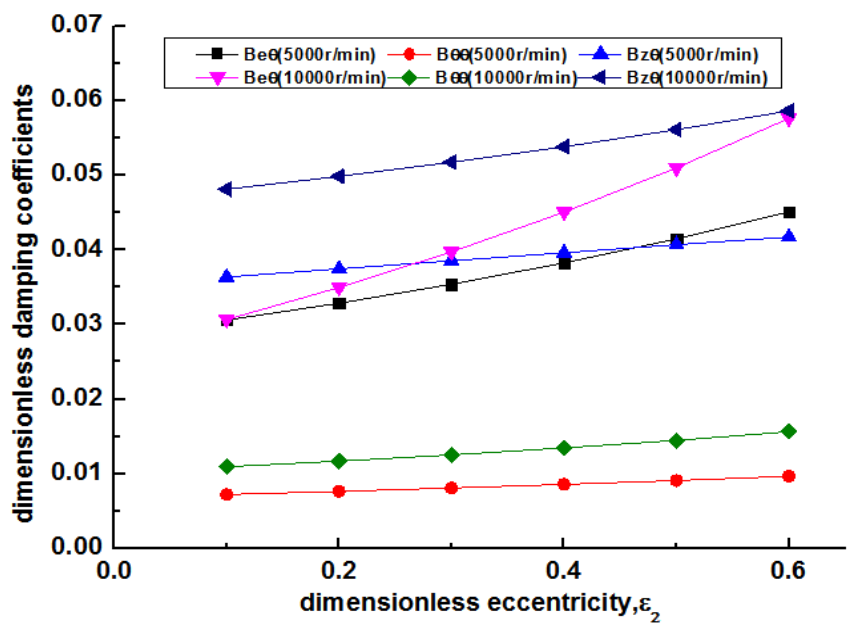

(b) $K_{e \theta}, K_{\theta \theta}, K_{z \theta}$

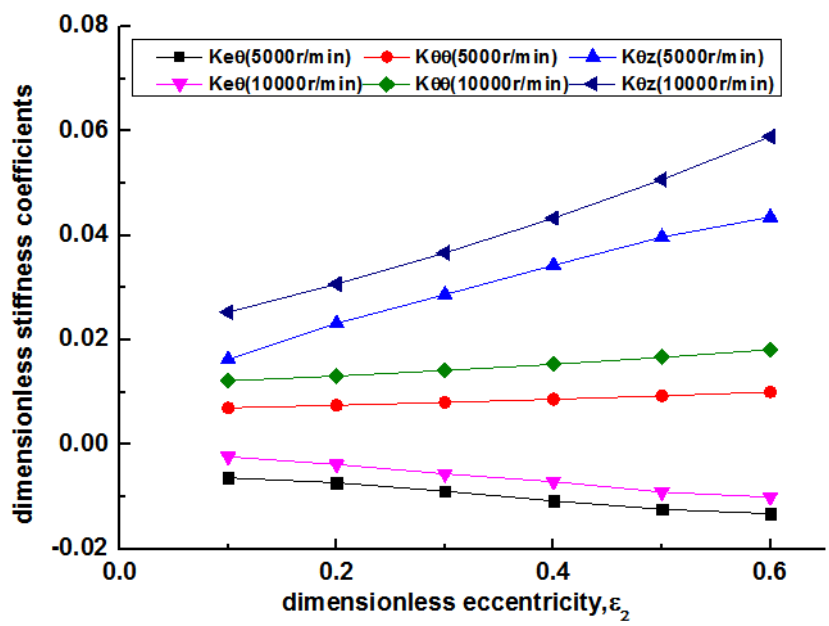

(d) $B_{e e}, B_{\theta e}, B_{z e}$

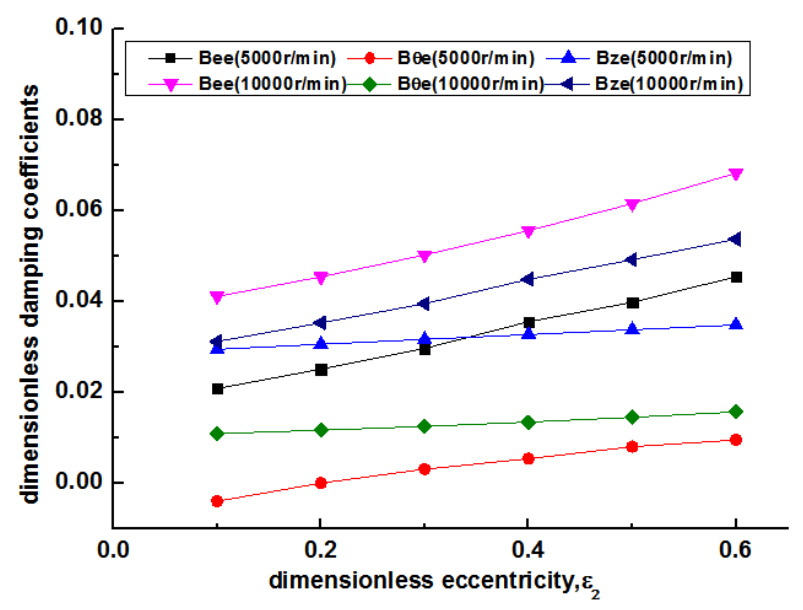

(d) $B_{e z}, B_{\theta z}, B_{z z}$

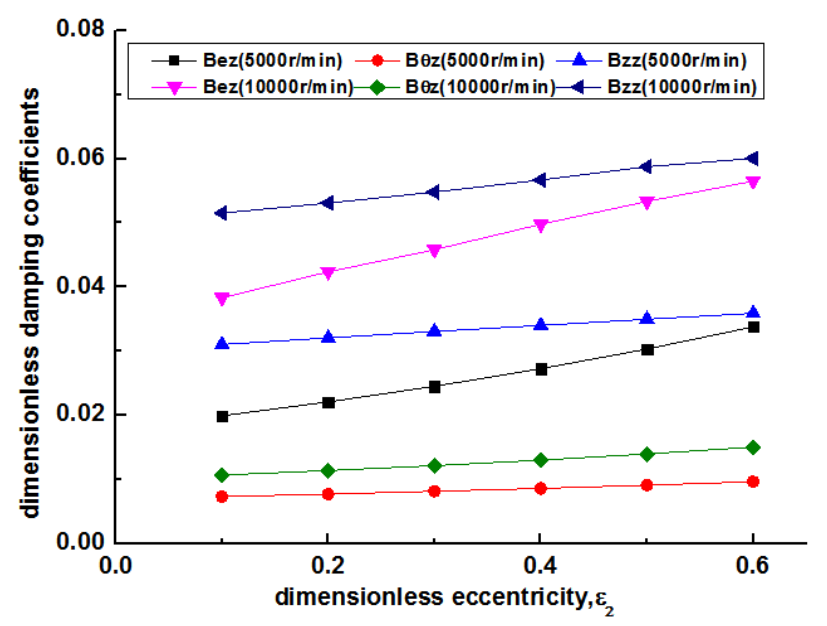

Fig. (5). Variation law of the calculated values of dynamic characteristic coefficients with speed $n$ and eccentricity $m$.

calculated values coincide with the experimental values, which validates the theoretical analysis and calculation.

\section{ANALYSIS OF SYSTEM STABILITY}

\subsection{The Stability Criterion}

Based on the assumption of small perturbation, the rotor deviates from the steady-state position by whirling lightly. The dynamic characteristic coefficients are the constants 
(a) $K_{e e}$

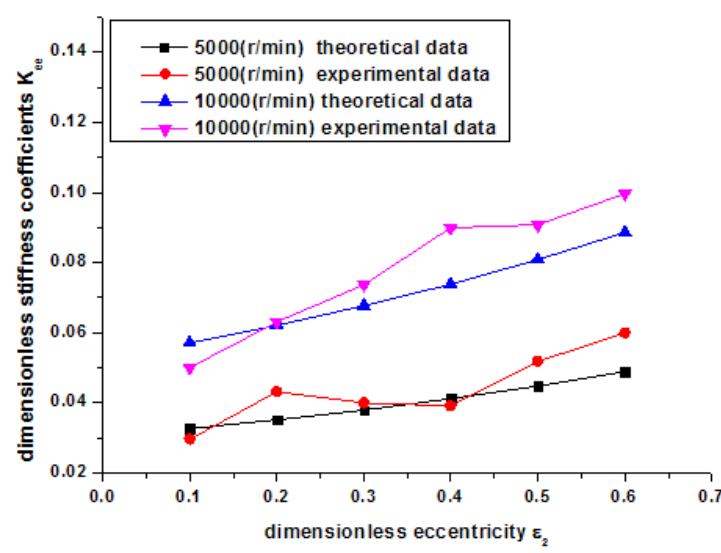

(c) $K_{z z}$

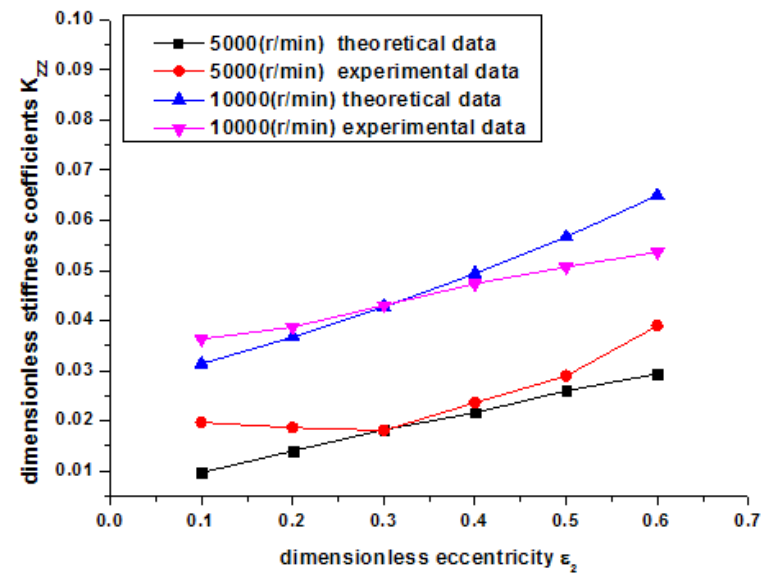

(e) $B_{\theta \theta}$

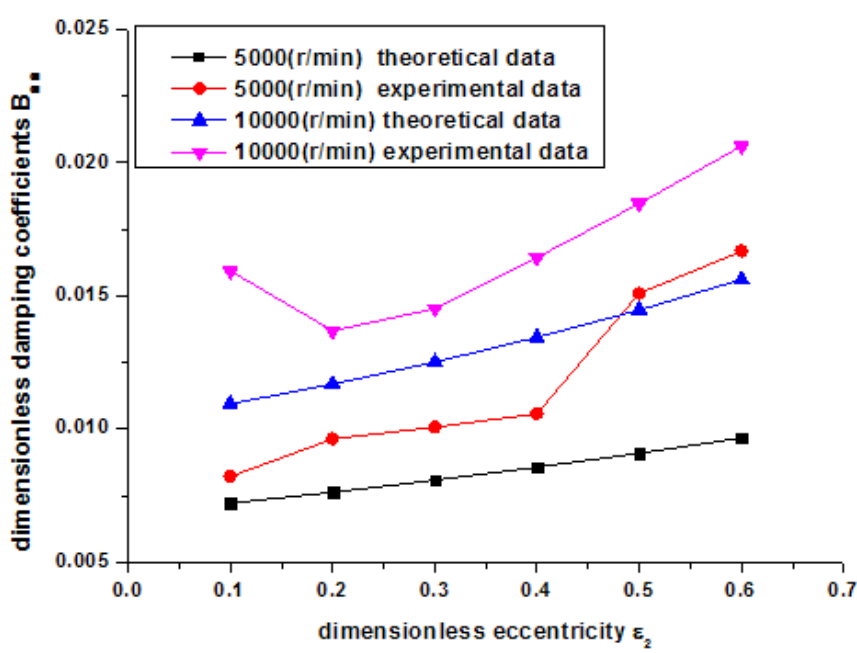

(b) $K_{\theta \theta}$

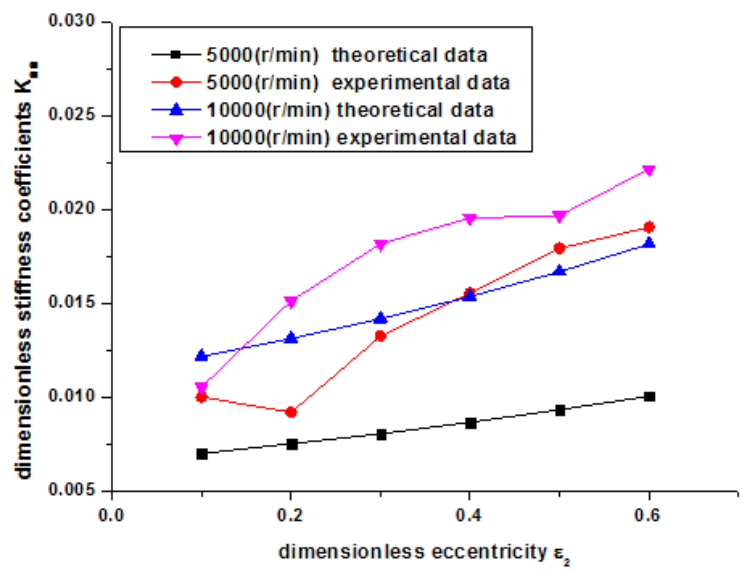

(d) $B_{e}$

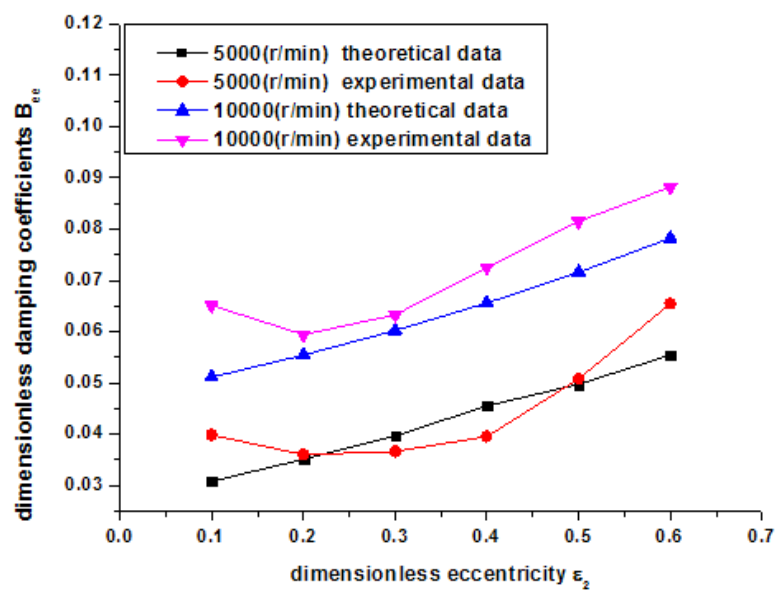

(f) $B_{z z}$

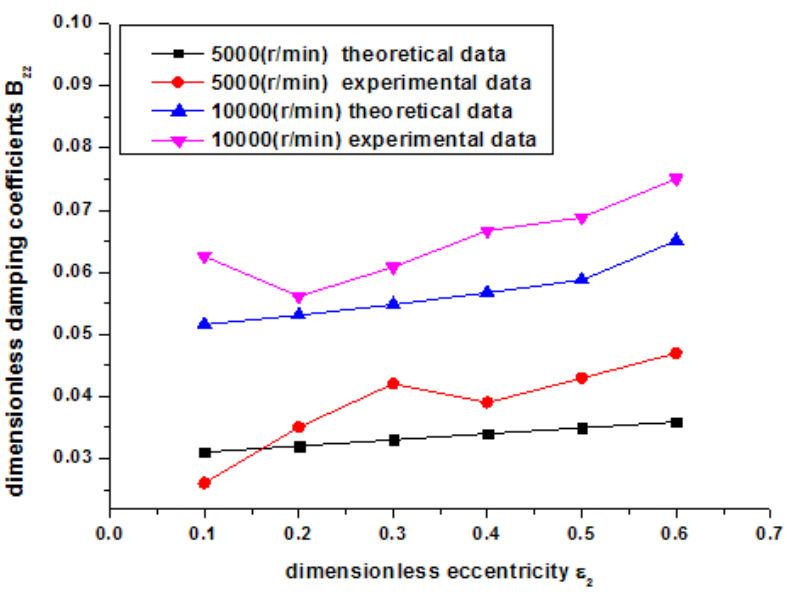

Fig. (6). Variation law of experimental values of dynamic characteristic coefficients with speed $n$ and eccentricity $\varepsilon_{2}$.

related to the structural parameters and the operation parameters.

The motion equation of the rotor can be derived:

$$
\left\{\begin{array}{c}
m e^{\prime \prime}+\Delta F_{\xi}=0 \\
m \theta^{\prime \prime}+\Delta F_{\eta}=0 \\
m z^{\prime \prime}+\Delta F_{z}=0
\end{array}\right.
$$


The functions of the gas film force increment and the operation parameters can be expressed as (by Taylor expansion):

$$
\left\{\begin{array}{c}
\Delta F_{\xi}=k_{e e} \Delta e+k_{e \theta} e \Delta \theta+k_{\theta z} \Delta z+b_{e e} \Delta e^{\prime}+b_{e \theta} e \Delta \theta^{\prime}+b_{e z} \Delta z^{\prime} \\
\Delta F_{\eta}=k_{\theta e} \Delta e+k_{\theta \theta} e \Delta \theta+k_{\theta z} \Delta z+b_{\theta e} \Delta e^{\prime}+b_{\theta \theta} e \Delta \theta^{\prime}+b_{\theta z} \Delta z \\
\Delta F_{z}=k_{z e} \Delta e+k_{z y} e \Delta \theta+k_{z z} \Delta z+b_{z e} \Delta e^{\prime}+b_{z \theta} e \Delta \theta^{\prime}+b_{z z} \Delta z^{\prime}
\end{array}\right.
$$

Eq. (7) can be simplified as:

$$
\left\{\begin{array}{c}
m e^{\prime \prime}+k_{e e} \Delta e+k_{e \theta} e \Delta \theta+k_{\theta z} \Delta z+b_{e e} \Delta e^{\prime}+b_{e \theta} e \Delta \theta^{\prime}+b_{e z} \Delta z^{\prime}=0 \\
m \theta^{\prime \prime}+k_{\theta e} \Delta e+k_{\theta \theta} e \Delta \theta+k_{\theta z} \Delta z+b_{\theta e} \Delta e^{\prime}+b_{\theta \theta} e \Delta \theta^{\prime}+b_{\theta z} \Delta z^{\prime}=0 \\
m z^{\prime \prime}+k_{z e} \Delta e+k_{z \theta} e \Delta \theta+k_{z z} \Delta z+b_{z e} \Delta e^{\prime}+b_{z \theta} e \Delta \theta^{\prime}+b_{z z} \Delta z^{\prime}=0
\end{array}\right.
$$

The general form of the solution can be expressed as:

$$
\left\{\begin{array}{c}
\Delta e=\xi_{0} e^{s t} \\
e \Delta \theta=\eta_{0} e^{s t} \\
\Delta z=Z_{0} e^{s t}
\end{array}\right.
$$

where, $\xi_{0} 、 \eta_{0}$ and $z_{0}$ are complex amplitude, $s$ is eigenvalue $(s=u+\omega i)$.

$$
\left\{\begin{array}{c}
\left(m s^{2}+b_{e e}+k_{e e}\right) \xi_{0}+\left(b_{e \theta} s+k_{e \theta}\right) \eta_{0}+\left(b_{e z} s+k_{e z}\right) Z_{0}=0 \\
\left(b_{\theta e} s+k_{\theta e}\right) \xi_{0}+\left(m s^{2}+b_{\theta \theta}+k_{\theta \theta}\right) \eta_{0}+\left(b_{\theta z} s+k_{\theta z}\right) Z_{0}=0 \\
\left(b_{z e} s+k_{z e}\right) \xi_{0}+\left(b_{z \theta} s+k_{z \theta}\right) \eta_{0}+\left(m s^{2}+b_{z z}+k_{z z}\right) Z_{0}=0
\end{array}\right.
$$

The matrix form can be written as:

$$
\left[\begin{array}{ccc}
m s^{2}+b_{e e}+k_{e e} & b_{e \theta} s+k_{e \theta} & b_{e z} s+k_{e z} \\
b_{\theta e} s+k_{\theta e} & m s^{2}+b_{\theta \theta}+k_{\theta \theta} & b_{\theta z} s+k_{\theta z} \\
b_{z e} s+k_{z e} & b_{z \theta} s+k_{z \theta} & m s^{2}+b_{z z}+k_{z z}
\end{array}\right]\left[\begin{array}{l}
\xi_{0} \\
\eta_{0} \\
Z_{0}
\end{array}\right]=\left[\begin{array}{l}
0 \\
0 \\
0
\end{array}\right]
$$

Due to the whirling of the bearing, the complex amplitude is not equivalent to 0 . The coefficient determinant of Eq. (10) can be expressed as:

$$
\left|\begin{array}{ccc}
m s^{2}+b_{e e}+k_{e e} & b_{e \theta} s+k_{e \theta} & b_{e z} s+k_{e z} \\
b_{\theta e} s+k_{\theta e} & m s^{2}+b_{\theta \theta}+k_{\theta \theta} & b_{\theta z} s+k_{\theta z} \\
b_{z e} s+k_{z e} & b_{z \theta} s+k_{z \theta} & m s^{2}+b_{z z}+k_{z z}
\end{array}\right|=0
$$

The characteristic equation is obtained by the determinate expansion:

$a_{0} s^{6}+a_{1} s^{5}+a_{2} s^{4}+a_{3} s^{3}+a_{4} s^{2}+a_{5} s^{1}+a_{6}=0$

Based on the stability theory, the stability of the system depends on the distribution of the $s$ in the complex plane. When $u<0$, the system is in the steady-state. If the rotor deviates from the steady-state position due to external perturbation, the amplitude of free oscillations decreases gradually with time. When $u=0$, the system is in the critical state. If the rotor deviates from the steady-state position, it will have sustained oscillation in the steady-state position. Its amplitude is determined by the initial conditions. When $u>0$, the system is in the unstable state. If the rotor deviates from the steady-state position, the trajectory will be divergent and the amplitude will increase gradually with time [13].

\subsection{Stability Analysis}

By programming the dynamic simulation and solving the real part $u$ of the eigenvalue $s$, the influence law of the speed and eccentricity on the system stability is studied. The bearing parameters and the operation parameters are shown in Table 1.

Fig. (7) shows the variation law of the real part $u_{\text {with the }}$ speed $(3000<n<25000 \mathrm{r} / \mathrm{min})$ and the eccentricity $\left(0.1<\varepsilon_{2}<0.6\right)$ :

1) The real part $u$ of the eigenvalue $S$ approaches zero with the increase of the speed $n$ and the stability of the bearing is close to the critical state. The system stability in the low speed range is better than the stability in the high speed range. The stability decreases gradually with the increase of the speed $n$.

2) The real part $u$ of the eigenvalue ${ }^{S}$ decreases with the increase of the eccentricity $\mathcal{E}_{2}$. Therefore, the system stability is better in the large eccentricity $\mathcal{E}_{2}$, and the operation of the bearing-rotor system is more stable.

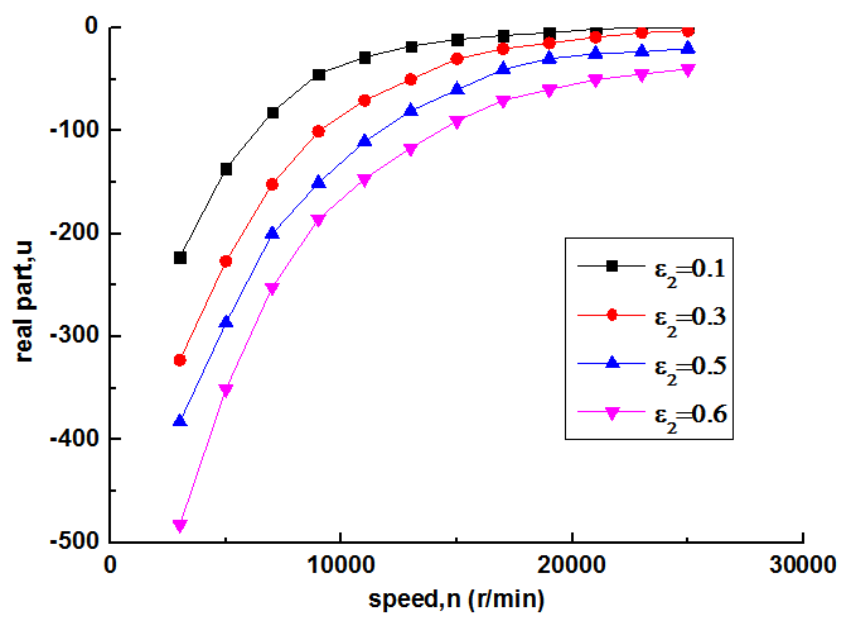

Fig. (7). Variation of the real part $u$ with speed $n$ and eccentricity $\varepsilon_{2}$.

\section{CONCLUSION}

1) The dynamic Reynolds equation is the basic equation for solving the dynamic characteristic coefficients. The correct solution of the equation is the basis for analyzing the bearing's dynamic characteristics and stability. Using the finite difference method, the lubrication problems can be solved accurately within less computing time and with more simplicity and it 
Table 1. Structure parameters.

\begin{tabular}{|c|c|c|c|c|c|c|c|c|c|}
\hline$r_{1}^{\prime}(\mathrm{mm})$ & $r_{g}^{\prime}(\mathrm{mm})$ & $r_{2}^{\prime}(\mathrm{mm})$ & $h_{0}(\mu \mathrm{m})$ & $\alpha$ & $\beta$ & $N g$ & $\bar{\delta}$ & $\bar{b}$ & $\varepsilon_{1}$ \\
\hline \hline 10 & 13 & 20 & 10 & 15 & 80 & 5 & 3 & 0.6 & 0.2 \\
\hline
\end{tabular}

contributes to analyzing the law of the bearing's dynamic characteristics and stability.

2) The increase of the bearing's speed contributes to improve the dynamic characteristic coefficients while the system stability approaches the critical state. The system stability in the low speed range is better than the stability in the high speed range.

3) With the increase of the eccentricity, the stiffness coefficients increase, while the damping coefficients increase eases up. The system stability approaches the steady-state with the increase of the eccentricity.

4) The influence of the dynamic characteristic coefficients on the stability of the bearing-rotor system depends on the comprehensive effect of stiffness and damping. Improving the speed and eccentricity contributes to the increase of the damping coefficients. The damping coefficients is an inhibitive factor for the whirling; it can consume the whirl energy and improve the system stability. But the stability of the bearing-rotor system approaches a critical state with the increase of the speed, which is a factor for the system instability. The eccentricity contributes to inhibit the whirling, but it can generate collision and friction to damage the bearing. Therefore, a reasonable choice of design parameters contributes to improving the bearing's comprehensive properties.

\section{CONFLICT OF INTEREST}

The authors confirm that this article content has no conflict of interest.

\section{ACKNOWLEDGEMENTS}

This project is supported by National Natural Science Foundation of China (Grant No. 51475142, 51275155), and Program for Innovative Research Team (in Science and
Technology) in University of Henan Province (Grant no: 13IRTSTHN025), and the Education Department Henan Province Science and Technology Research Projects (Grant no: 13A460251).

\section{REFERENCES}

[1] W. YunFei, "Gas lubricated theory and design manual of gas bearings", Beijing: China Machine Press, 1999.

[2] Z. Guang, Y. Hechun, and M. Wenqi, "Review of research on rotor-gas bearing-flexible support system", Lubrication Engineering, vol. 35, 115-122, 2010.

[3] Y. Pan, Z. Keqin, and W. Xiaoli, "On the non-linear stability of self-acting gas journal bearings", Tribology International, vol. 42, 71-6, 2009

[4] Z. Yongfang, Z. Shisheng, and L. Yanjun, "The behavior of gaslubricated of axial-groove sliding bearing-rotor", Journal of Vibration, Measurement \& Diagnosis, vol. 33, 220-23, 2013.

[5] Z. Guang-hui, and L. Zhan-sheng, "Dynamic study of journal gas bearings-flexible rotor coupling system", Journal of Aerospace Power, vol. 25, 1417-26, 2010.

[6] K. Wei, and Z. Jia-zhong, "Stability and bifurcation of symmetrical rotor in self-acting gas journal bearings", Journal of Aerospace Power, vol. 22, 1537-43, 2007.

[7] M. Arghir, and P. Matta, "Compressibility effects on the dynamic characteristics of gas lubricated mechanical components", Comptes Rendus Mécanique, vol. 337, 739-747, 2009.

[8] J. Schiffmann, and D. Favrat, "The effect of real gas on the properties of Herringbone Grooved Journal Bearings“", Tribology International, vol. 43, 1602-14, 2010.

[9] L. Zhiwei, W. Mingming, and L. Bo, "Analysis on the gas pressure distribution of conical spiral groove aerodynamic bearings", Journal of Xi'an Technological University, Vol. 28, 224-28, 2008.

[10] J. Chenhui, Y. Wei, and Q. Ming, "Static characteristics analysis of conical spiral groove aerodynamic bearings", Lubrication Engineering, vol. 2, 14-19, 2013.

[11] Z. Quan, Z. Xiangxiong, and C. Rugang, "Static characteristic analysis of aerodynamic gas thrust bearing based on finite difference method". Lubrication Engineering, vol. 34, 6-9, 2009

[12] J. Liu, and M. Yoshihiro, "Analysis of oil-lubricated herringbone grooved journal bearing with trapezoidal cross-section, using a spectral finite difference method“, Journal of Hydrodynamics, vol. 22, 408-412, 2010.

[13] Y. Lie, and L. Heng, "Dynamics of Bearing-Rotor System", Xi An: Xi'an Jiao Tong University press, 2001. 